\title{
Wussten Sie schon ...
}

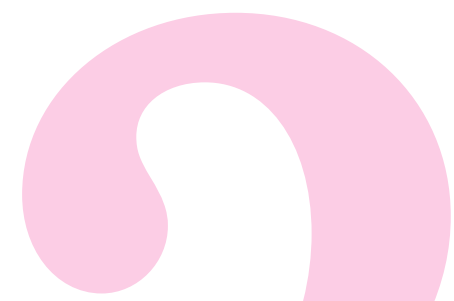

\section{... dass starker Kaffeekonsum im ersten Trimester wahrscheinlich doch mit einem erhöhten Abort- risiko verbunden ist?}

Eine Forschergruppe in Schweden verglich 652 Frauen mit Spontanaborten zwischen der 6 . und 12. Schwangerschaftswoche mit 953 Schwangeren ohne Aborte (matched controls) [Cnattingius S et al: N Engl J Med 2000;343: 1839-1845]. Bei Nichtraucherinnen wurden Spontanaborte häufiger beobachtet, sofern diese mindestens 100 mg Koffein pro Tag (1 Tasse) konsumierten. Das Abortrisiko verdoppelte sich beim Genuss von $500 \mathrm{mg}$ Koffein pro Tag (5 Tassen) oder mehr.

\section{Kommentar}

Die Stärke dieser Studie war sicher, dass bei allen Frauen die Nikotinkonzentration gemessen wurde und eine Karyotypisierung des Abortmaterials erfolgte. Der dosisabhängige Zusammenhang zwischen Koffeineinnahme und Abortrisiko betraf wie erwartet nur Nichtraucherinnen und solche, bei denen die Karyotypisierung des Abortmaterials normal war. Der beobachtete Zusammenhang gibt uns wieder etwas auf die immer länger werdende Liste von sinnvollen Verhaltensänderungen für Frauen zur Optimierung einer Schwangerschaft.

$m . k . h$.
... dass Agnus-castus-Extrakt zur Behandlung des prämenstruellen Syndroms wirksam ist?

In einer grösseren rigorosen und methodisch guten randomisierten plazebokontrollierten Doppelblindstudie mit 170 Frauen war Agnus-castus-Extrakt (Vitexagnus-castus-L-Extrakt Ze 440; Premens, Prefemin ${ }^{\circledR}$ ) statistisch hochsignifikant wirksamer als Plazebo. Dies betraf die Selbstbeurteilung (mittels VisualAnalog-Skala) von Reizbarkeit, Stimmungsalterationen, Wut, Kopfschmerz, Brustspannung, nicht aber Aufgetriebensein. Das Präparat hatte keine nennenswerten Nebenwirkungen [Schellenberg R: Br Med J 2001; 322:134137].

\section{Kommentar}

Nachdem pflanzliche Mittel heute im allgemeinen eine höhere Akzeptanz als «Chemie» (sic!) haben, erweitert Agnus-castusExtrakt unser therapeutisches Armentarium dieses leidigen und häufigen Problems, das vor allem Frauen in der zweiten Hälfte des 3. und ersten Hälfte des 4. Lebensdezenniums betrifft, um so mehr als viele der empfohlenen Substanzen nach einigen Monaten nicht mehr so wirksam sind wie zu Beginn.

$m \cdot k \cdot h$
... dass Östrogene eine positive Wirkung auf die Stimmbänder entfalten?

Im Rahmen einer Fallkontrollstudie wurden 48 prämenopausal adnexektomierte Frauen, welche transdermale Östrogene erhielten, mit solchen ohne Hormonersatz verglichen [Caruso $\mathrm{S}$ et al: Fertil Steril 2000;74:1073-1079]. Die zytologischen Abstriche aus Vagina und Larynx waren sich sehr ähnlich, d.h. ohne Hormonersatz dominierten Atrophie und Dystrophie, mit Östrogenen jedoch Superfizialzellen. Die subjektiv empfundene Stimmqualität war statistisch signifikant besser unter Östrogentherapie.

\section{Kommentar}

Bewusst wurden nur ovarektomierte Frauen untersucht, da Androgene, welche zu typischen Stimmveränderungen führen (Unregelmässigkeit der Stimme, rascher Timbrewechsel, Erniedrigung der Basisfrequenz), ja auch in der normalen Menopause während einer gewissen Zeit von den Ovarien produziert werden. Die Studie belegt, dass der Larynx eindeutig ein Östrogenzielorgan ist. Genauso wie sie dies an der Vagina tun, können Östrogene offenbar auch das Stimmband vor dystrophischen Veränderungen schützen. Wieder einmal ein Argument pro Hormonersatztherapie.

m.k.h 
... dass Belegarzttätigkeit der gewichtigste Faktor ist, wenn es um die Episiotomieinzidenz geht?

Bei 1576 konsekutiven Spontangeburten bei Primigravidae am Brigham and Women's Hospital in Boston wurde die Episiotomierate in Bezug zu demographischen und geburtshilflichen Faktoren gesetzt [Obstet Gynecol 2000;96: 214]. Insgesamt betrugt die Episiotomierate $40,6 \%$. Hebammen hatten eine statistisch signifikant geringere Episiotomierate $(21,4 \%)$ als Spitalärzte $(33,3 \%)$ und Belegärzte $(55,6 \%)(\mathrm{p}=0,001)$. Die höchste Voraussagekraft für die Durchführung einer Episiotomie war der Status eines Belegarztes (OR 4,1), gefolgt von Spitalarzt (OR 1,7), verlängerte Austreibungsperiode (OR 1,8), fetale Makrosomie $\geq 4000 \mathrm{~g}($ OR 1,6$)$ und Epiduralanalgesie (OR 1,4). Entsprechend der in den USA geübten Praxis der medianen Episiotomie, welche per se mit einem erhöhten DR-III- bzw. DR-IVRisiko einhergeht, war deren Rate bei Hebammen mit 5,3\% am niedrigsten. Belegärzte hatten eine beinahe doppelt so hohe Rate mit 10,1\%, bei den Spitalärzten betrug sie $11,5 \%$.

\section{Kommentar}

Überflüssig!
... dass es einen erheblichen Unterschied macht, ob bei der ersten Pränatalvisite das Patientinnengespräch von einem Frauenarzt oder von einer Frauenärztin geführt wird?

In einer Vergleichsuntersuchung zwischen männlichen und weiblichen Frauenärzten wurden die ersten Pränatalvisiten per Tonband aufgezeichnet und im Anschluss hinsichtlich ihrer kommunikativen, sachbezogenen und emotionalen Inhalte ausgewertet, bezogen auf die Rezeption der Patientin [Obstet Gynecol 1999; 93:635]. Männer machten 30\% mehr Feststellungen und hatten eine längere Gesprächszeit. Diese ergab sich dadurch, dass Patientinnen mehr Gesprächszeit in Anspruch genommen hatten, wenn sie mit einem Gynäkologen konfrontiert waren. Gerade umgekehrt verhielt es sich, wenn es um die emotionale Empfindsamkeit der Betreuenden ging. Hier schnitten Frauen ebenso besser ab wie im Bereich der informationsbezogenen Partnerschaftlichkeit. Wenn es um die medizinische Handlung, also um die Untersuchung selbst ging, wurden Frauenarzt und Frauenärztin gleich bewertet.

\section{Kommentar}

b.s. $\quad$ Diese Studie räumt zumindest mit einem Vorurteil auf, nämlich dass die Gynäkologen sich weniger Zeit für ihre Patientinnen nehmen. Aus männlicher Sicht frustriert diese Studie insofern, als das längere Gespräch mit der Patientin offensichtlich nicht honoriert wird, zeigt die Studie doch, dass keine der Zufriedenheitsdimensionen sich mit der Dauer des Gesprächs korrelieren liess. Die bessere Akzeptanz der Gynäkologin gerade bei der jungen Frau hat die Pharmaindustrie beispielsweise im hart umkämpften Pillenmarkt schon vor längerer Zeit erkannt. So kann man beispielsweise anhand einer Pilleneinsteiger-Broschüre mit mehrseitigem Text und einigen Karikaturen wahrnehmen, dass - weder in Wort noch in Bild - kein einziges Mal der Frauenarzt erwähnt wurde. Vielleicht ist es an der Zeit, sich mit dem Thema "gender discrimination» einmal auseinander zu setzen. Ob es denn hilft?

... dass zwei Patienten mit einer frontotemporalen Demenz plötzlich ein Faible für Pop-Musik entwickelten?

Frisoni et al. [Neurology 2000;55: 1935] berichten über zwei Patienten: Einer konnte früher Pop-Musik nicht ausstehen, der andere hatte überhaupt kein Interesse für Musik. Die Forscher spekulieren, dass es zu Schäden in Hirnverbindungen gekommen war, die für 
Ton, Höhe und Rhythmus und Vertrautheit verantwortlich sind.

\section{Kommentar}

Die Forscher betonten, dass dies aber nicht heisse, dass ein $\mathrm{Zu}$ sammenhang bestehe zwischen Vorliebe für Pop-Musik und einem frontalen Hirnschaden.

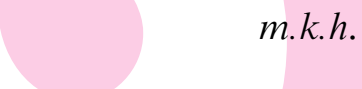

... dass nosokomiale Blutinfektionen die achthäufigste Todesursache in den USA sind und dass häufig antibiotikaresistente Keime im Spiel sind?

Die drei häufigsten Erreger bei positiven Blutkulturen sind koagulasenegative Staphylokokken ( $80 \%$ davon sind methicillinresistente Stämme), Staphylococcus aureus (30\% der Stämme methicillinresistent) und Enterokokken (20\% der Stämme sind vancomycinresistent) [Wenzel RP, Edmund MB: N Engl J Med 2000;343: 1961-1963]. Beunruhigend ist auch die steigende Zahl von Streptokokkenpneumoniefällen, die gegen viele Antibiotika resistent sind. Die Autoren des dazugehörigen Editorials im N Engl J Med weisen auf den «antibiotic pressure» hin (Verhältnis von Antibiotikaverbrauch und Selektion resistenter Keime). In den USA berechnet man 30 Antibiotikaverschreibungen pro 100 Personen/Jahr oder 4,1 kg Antibioti- ka pro 100 Personen/Jahr. Von den 25000 Tonnen Antibiotika, die in den USA pro Jahr verschrieben werden, fallen 50\% auf Menschen und 50\% auf Tiere, Landwirtschaft und Aquakulturen. Etwa die Hälfte der Verschreibungen sei unnütz (z.B. virale Infektion im HNO-Bereich, Bronchitis und Pharyngitis).

\section{Kommentar}

Daten aus Ungarn und Island belegen, dass ein abnehmender Penicillinverbrauch einhergeht mit einer Abnahme der penicillinresistenten Pneumokokkenstämme. Neben einem restriktiven Einsatz von Antibiotika weisen die Autoren auf weitere Massnahmen hin, um das Problem in den Griff zu bekommen (bessere Hygiene im Spital, bessere diagnostische Schnelltests, Pneumokokkenimpfung HIV-positiver Menschen). m.k.h.

... dass Sojaproteine (gehören zu den Phytoöstrogenen) im Vergleich zu Plazebo weder Libido, vasomotorische Symptome (Wallungen), psychologische Symptome noch vaginale Trockenheit beeinflussen?

Die doppelblinde plazebokontrollierte Studie [Kotsopoulos D et al: Climacteric 2000;3:161-167] mit relativ hohen Mengen von Sojasupplementen betraf 97 postmenopausale Frauen im Alter zwischen 50 und 75 Jahren. Zwei weitere randomisierte plazebokontrollierte Studien mit Promensil, einem Extrakt aus rotem Klee (Trifolium pratense) [Knight DC et al: Climacteric 1999,2:79_ 84; Baber RJ et al: Climacteric 1999;2:85-92], welche die Wirkung dieses isoflavonhaltigen Naturmittels auf Wallungen testeten, kamen ebenfalls zu einem negativen Resultat.

\section{Kommentar}

Phytoöstrogene sind nichtsteroidale Substanzen, die eine östrogene Aktivität haben oder in Substanzen metabolisiert werden, welche östrogene Wirkungen entfalten. Phytoöstrogene werden in drei Gruppen klassifiziert: Isoflavone, Cumestane und Lignane. Sojabohnen sind eine reichhaltige Quelle für Phytoöstrogene, welche vor allem Isoflavone enthalten. Isoflavone sind dadurch charakterisiert, dass abhängig von der gastrointestinalen Mikroflora die Absorption variiert und die Bioverfügbarkeit somit ebenfalls. Die vorhandenen Daten zeigen eindeutig, dass Sojaproteine und Isoflavone keinen Einfluss auf Wallungen haben. Wenn Patientinnen über eine positive Wirkung berichten, handelt es sich um einen Plazeboeffekt.

m.k.h 